\title{
Preparar al maestro para el cambio educativo
}

\section{Preparing teachers for educational change}

DOI: $10.46932 / \mathrm{sfjdv2n3-049}$

Received in: May 1st, 2021

Accepted in: Jun 30th, 2021

\author{
Lesbia Gertrudis Cánovas Fabelo \\ Grado científico \\ Doctor en Ciencias Filosóficas Centro: Asociación de Pedagogos de Cuba \\ País de procedencia: República de Cuba \\ Dirección particular: Calle 84 No. 502 apto 214 e/ 5ta y 5ta A, Playa, La Habana \\ Correo electrónico: lesbia@ rimed.cu
}

\section{RESUMEN}

Existe una toma de conciencia en relación con la necesidad de producir cambios en la educación; pero no está suficientemente claro por qué y para qué cambiar, qué, cómo y quién debe cambiar. Aparece siempre en el horizonte la amenaza de nuevos dogmatismos, y sobre todo de ejercicios tradicionales de cambio que son más deseos que realidades.

Repensar la formación de los maestros implica pensar en los contenidos de la formación, en los que enseñará, cómo los transmitirá, (incorporando las TIC), y el tipo de prácticas para trabajar en el aula, con la familia y la gestión en la escuela, pero también cómo debe ser el proceso formativo para que estén en capacidad de promover el cambio educativo.

El modelo de formación debe poner el énfasis en que el aprendizaje ocurre en una relación entre el profesor y los estudiantes, donde intervienen dos aspectos cruciales, uno es cognitivo: el conocimiento, dominio de la materia que enseña, y el otro es emocional: la capacidad para poder interpretar el mundo interno de los alumnos.

El Proyecto Con infinito amor pone el énfasis en propiciar la participación de los estudiantes, motivarlos de manera que sepan reflexionar y orientarse acertadamente ante su realidad.

\footnotetext{
ABSTRACT

There is a growing awareness of the need to bring about change in education, but it is not sufficiently clear why and what to change, what, how and who should change. The threat of new dogmatisms always appears on the horizon, and above all of traditional exercises of change that are more desires than realities. Rethinking teacher training implies thinking about the contents of the training, what they will teach, how they will transmit them (incorporating ICT), and the type of practices to work in the classroom, with the family and school management, but also what the training process should be like so that they are able to promote educational change.

The training model should emphasize that learning occurs in a relationship between the teacher and the students, where two crucial aspects are involved, one is cognitive: knowledge, mastery of the subject taught, and the other is emotional: the ability to interpret the inner world of the students.

The Con infinito amor project emphasizes the participation of students, motivating them so that they can reflect and orient themselves correctly in the face of their reality.
} 


\section{INTRODUCCIÓN}

Vivimos en un periodo de cambio de paradigma(s) mucho más evidente en las ciencias naturales, pero también en las ciencias sociales y humanísticas, lo cual influye y está influido,decisivamente, por la educación.

Una nueva teoría se manifiesta asociada a la resolución de problemas concretos de la realidad, de la práctica.

Los intensos debates que tienen lugar en la actualidad, en diversas ramas del saber, y particularmente en las Ciencias de la Educación, son expresión de los cuestionamientos que se hacen a los paradigmas vigentes, en diversos campos y especialidades científicas, por parte de sectores de la comunidad científica.

Del estudio de idénticas fuentes y el análisis de logros similares pueden surgir distintos paradigmas en profesionales del mismo, o de análogos, campos del saber. Esto constituyeun gran reto para el proceso de enseñanza y de aprendizaje, tan acostumbrado a trasmitir "verdades absolutas" y con validez universal.

Lo que sin dudas es necesario a la educación en el momento actual, es colocar al hombre mismo como problema, su humanización; hacer posible la praxis como reflexión y acción sobre el mundo para transformarlo (Freire).

La misión principal de la educación, en su más amplio sentido, es la de crear desde las primeras edades las condiciones propicias para el surgimiento en la juventud de una personalidad madura, que continúe potenciándose a lo largo de toda la vida de la persona.

El sistema educativo tiene que partir de una concepción filosófica que sirva de fundamento a las "verdades cognitivas y éticas", que inspiren la conformación de su funcionamiento y, en particular, del sistema de actividades y de relaciones en que tiene lugar la educación de los seres humanos y su relación con el contexto, con su realidad.

Las teorías deben servir como vías para conformar currículos que permitan acceder a explicaciones científicas sobre el mundo, dar acceso a los resultados de las revoluciones científicotécnicas, mostrando las incongruencias en su impacto en diferentes regiones y entre poderosos y desposeidos.

La ideología, en correspondencia con el sistema filosófico de partida, especialmente lapolítica, en tanto "concepción de la realidad antroposocial”, guiará la conexión de la educación con la "praxis política y social" y es muy necesaria porque en su núcleo se funden paradigmas/axiomas y valores, (cuidando no se mistifiquen).

Es importante asumir la educación desde una nueva visión de las emociones, por su papel en el 
encuentro de las personas con el mundo, su relación con el pensamiento, los valores, las necesidades, deseos, aspiraciones, temores, etc., aunque sean fuentes que alteran el equilibrio de las ideologías políticas.

\section{DESARROLLO:}

La dirección pedagógica del Proyecto “Con infinito amor”: La educación del carácter y los valores familiares, encamina su contribución a la formación del ser humano al que se aspira. Su visión es preparar a los adolescentes que se forman como maestros para que sean mejores personas, aprendan a involucrarse y comprometerse, lo que también se promueve entre los profesores. Coloca en primer plano al estudiante como persona humana de bien (buena persona) porque es su interés que esa sea la base que le sirva para el desempeño exitoso de su rol profesional como educador.

Con la labor educativa de los educadores y las instituciones educativas, entendida en su amplio sentido, se cumple el encargo social de contribuir de manera consciente y planificada a la formación de la personalidad de los niños, adolescentes y jóvenes. Guiada por el criterio fundamental de la diferencia y unidad de la personalidad y su medio externo socio histórico, de que ambos interaccionan y por esa vía se identifican, penetran y determinan recíprocamente puede comprenderse la función activa y creadora de la subjetividad humana,tan importante para el proceso educativo.

El Proyecto se ha propuesto acompañar a los estudiantes en su activa incorporación al reconocimiento de quiénes y cómo son, de dónde vienen y hacia dónde quieren y deben dirigir su futuro, desde el presupuesto vigotskiano de que la educación es la que guía el desarrollo, lo precede y conduce.

\section{LOS ESTUDIANTES DE LAS ESCUELAS PEDAGÓGICAS}

Los adolescentes que ingresan a estas instituciones han concluido el 9no. grado. Unasignificativa parte de ellos procede de familias monoparentales o reconstruidas con jefatura femenina, donde sus madres asumen todas las responsabilidades, como resultado de divorcios o de la no implicación de la figura paterna en las dinámicas principales.

La familia es una institución que ejerce una influencia decisiva en los principales procesos enque estos adolescentes se involucran, pero no está exenta de vivencias poco desarrolladoras, sobre todo vinculadas a las dificultades para establecer límites y horarios, el desarrollo de habilidades para la resolución de conflictos e instaurar una comunicación que trascienda la transmisión de información y la regulación de la conducta de los hijos.

Entre las principales preocupaciones y conflictos que mayoritariamente plantean están las relaciones con su familia, a pesar de ello, valoran altamente su importancia. 
En su condición de adolescentes, en ellos se han venido produciendo cambios biológicos y fisiológicos que impactan en su subjetividad y comportamiento, manifestándose especialmente en la esfera de la sexualidad.

A pesar del trabajo que se realiza en las escuelas de los niveles precedentes para la educación de la sexualidad sana y responsable, casi la totalidad al ingresar a estos centros, ya inició las relaciones sexuales e incurren en cambios relativamente frecuente de parejas. Por otra parte, sobre esta esfera manifiestan desconocimiento y confusiones, además del inadecuado uso de los medios de protección y los métodos anticonceptivos. Esta es una de las problemáticas que más despierta su interés y en la que se sienten menos comprendidos yorientados por sus familiares y profesores.

En su sistema de relaciones se reafirma la importancia de los coetáneos en las dinámicas cotidianas del grupo escolar, aunque en este ámbito es frecuente la existencia de conflictos que tampoco saben afrontar y en la que consideran que tampoco son comprendidos y apoyados por sus profesores.

Otra esfera donde mayoritariamente plantean insatisfacción es en las actividades culturales yla recreación. Lo que más les gusta es compartir con sus amigos, con su pareja; ver novelas,películas, escuchar música (sobre todo reguetón y romántica); ir a fiestas, (sobre todo en centros recreativos), a la playa, piscina o ríos, jugar y ver productos audiovisuales. Los varones también incluyen entre sus preferencias el deporte y los videojuegos. Entre sus deseos muy pocas veces aparece algo que contribuya a su desarrollo intelectual o espiritual, ni relacionado con su preparación para la vida adulta o profesión.

Aunque reconocen la labor educativa que se realiza en sus escuelas, no encuentran en ellas una plena satisfacción. Consideran que en algunos profesores existe falta de ejemplaridad y manejo inadecuado en situaciones de indisciplina. Tampoco siente satisfacción con todas lasasignaturas que reciben y en algunos casos no sienten que les aportan mucho.

No siempre se sienten respetados ni que se toman en cuenta sus opiniones. Además, consideran que les dan poca participación en la toma de decisiones.

Generalmente sus resultados académicos en el nivel precedente no son de los mejores. Están motivados por el uso de las TIC, aunque no todos pueden acceder a ella, lo cual esuno de sus principales deseos; de ahí que también encuentren ahí una de lasinsatisfacciones que tienen con la escuela. Aprecian que los recursos tecnológicos y el nivel de acceso a las TIC contribuyen a la aceptación en el grupo de coetáneos y lo asocian a un mejor estatus social.

Los deseos que predominan en la mayoría de estos estudiantes son de obtención de bienes materiales, sobre todo asociados a las TIC, otra parte son de placer personal, como viajar, y en una minoría están relacionados con el estudio y la profesión.

Pertenecen a la Federación de Estudiantes de la Enseñanza Media, pero no consideran que existe 
un real protagonismo estudiantil y su papel es limitado. Rechazan las actividades formales, monótonas, que no le despiertan el interés porque no toman en cuenta sus preferencias e intereses y son tradicionalistas.

Aunque haberse incorporado a la escuela pedagógica, constituye un paso decisivo en la definición de su futura profesión, no significa que en todos ellos exista vocación para el magisterio. Lograr que se desarrolle depende de la labor mancomunada y coherente entre la escuela formadora y las instituciones educativas donde realizan la práctica laboral. Nosiempre las escuelas donde son ubicados tienen las condiciones necesarias para servir de modelo a los estudiantes en formación, ni todos los tutores designados están en condiciones de guiarlos en la solución de los problemas profesionales que deben aprender a resolver enla vinculación de la teoría con la práctica.

La eficiencia en el ciclo de las 4 graduaciones se encuentra en el rango de 60,2\% y 64,6\%, hay un discreto incremento, pero es baja y no satisface las necesidades. La permanencia de los que se incorporaron a los estudios superiores es de un $42 \%$.

Desde su ingreso a las escuelas pedagógicas el centro de atención se coloca en la formaciónde esos adolescentes como futuros profesionales de la educación. Su ejercitación como maestros y educadores se incrementa progresivamente durante la formación de nivel medioy se focaliza su preparación para continuar estudios superiores. Sin embargo, es insuficiente la prioridad que se confiere al conocimiento de sí mismos, de sus conflictos, necesidades e intereses de todo tipo, a la reflexión sobre su cotidianidad de manera realista, especialmente cuando para una gran mayoría, ese espacio tan necesario tampoco existe en sus hogares.

En los documentos normativos del MINED se define que:

\begin{abstract}
"El fin de la escuela pedagógica lo constituye la formación integral de la personalidad de cada estudiante entre 14 y 18 años, mediante la ampliación, aplicación y profundización de los contenidos, en correspondencia con los ideales patrióticos y humanistas de la sociedad socialista cubana en su desarrollo próspero y sostenible;

expresados en sus formas de sentir, pensar, actuar de manera independiente, de acuerdo con su nivel de desarrollo y particularidades individuales, intereses y necesidades sociales, que le permita la dirección del proceso educativo en las diferentes instituciones educacionales y modalidades educativas que atienden a educandos de la Primera Infancia, la Educación Primaria y la Educación Especial, paracontribuir a la educación integral de sus educandos con necesidades educativasasociadas o no a discapacidades", (MINED).
\end{abstract}

En el fin declarado, así como en los objetivos generales y por años no se dispone de un modelo que permita proyectar, en toda su complejidad, el desarrollo humano, a partir de las características reales de los estudiantes y no solo desde las aspiraciones. 


\section{CONCEPCIÓN CURRICULAR PARA SU FORMACIÓN}

El currículo está considerado "como el proyecto educativo integral con carácter de proceso, que expresa las relaciones de interdependencia en un contexto histórico-social, condiciónque le permite rediseñarse sistemáticamente en función del desarrollo social, progreso de la ciencia y necesidades de los estudiantes, que se traduzca en la educación de la personalidaddel ciudadano que se aspira a formar" (MINED, abril, 2018). La grada curricular que conformael plan de estudio (5 616 horas clases) está integrada por las asignaturas de:

Formación general que le proporcionan el nivel de Educación Media Superior y representa el $31 \%$ del fondo de tiempo total; para la preparación pedagógica y psicológica general, que representan el 6\% y las particulares de la especialidad dedicadas a las didácticas particulares de las asignaturas que se imparten en la Educación Primaria, además de la Literatura Infantil, representan el 13\%.

A los Talleres Profesionales se dedica el 3\% del fondo de tiempo total, dentro de ellos, la escuela pedagógica podrá dedicar 72 horas “....a la satisfacción de las necesidades que plantea el contexto” y el resto se emplea en 4to año en: Taller de textos martianos, Uso de los recursos audiovisuales e informáticos, además de Uso y cuidado de la voz del profesional. En el 4to. año, se dedican 240 horas clase (4\%) a la Preparación para la Culminación de estudios.

El Sistema de Formación Laboral constituye “...la columna vertebral del Plan de estudio alrededor del cual se integran todas las asignaturas y los diferentes procesos que tienen lugar en las instituciones" y se le asigna el $31 \%$ del fondo de tiempo total.

El 15\% (864) del total de horas se dedica a las Actividades complementarias y el Trabajo independiente y grupal, distribuidas a lo largo de 7 semestres, excepto el 8vo. grado (porque permanecen todo el tiempo en las escuelas), la cantidad de horas asignada es variada y no se precisa a partir de qué se define a qué se dedicarán, cómo se realizan, ni el nivel en que se decide.

Los problemas planteados por los estudiantes que fueron expuestos anteriormente, se corroboran por otros estudios e investigaciones, así como por otras fuentes, y nos alertan acerca de que la educación y la escuela están fallando en su función de preparación de los seres humanos para la vida. Las prácticas habituales van siendo inefectivas para los propósitos actuales de desarrollo personal y social.

Las exigencias contemporáneas conllevan a abandonar el enfoque centrado en el docente "poseedor de todo el saber y trasmisor de información”, para convertirse en conductor y guía,asumiendo tareas de impulso, ayuda, mediación, coordinación y dirección del aprendizaje; la formación y autoeducación del educando, entendida como "aprender a aprender", en su amplio sentido, que implica, como decía Carlos Marx que el educador también debe ser educado. De ahí que corresponde a la pedagogía: 
“...tomar como fuente un modelo interdisciplinario para lograr verdaderamente el desarrollo esperado de sus educandos. Ya no se trata de trasmitir fundamentalmente conocimientos, pues se requiere ser capaz para llevar a cabo el desarrollo sostenible de la sociedad. Esto implica que las personas tienen que saber sostener también su propio desarrollo" (Fariñas, 2009).

\section{LA ESENCIA DEL APRENDIZAJE OBTENIDO EN EL PROYECTO}

A continuación se exponen los resultados de la sistematización de una investigación acción participativa realizada en 2 etapas durante los cursos 2014-2016 y 2016-2018, que abarcó una muestra de 113 grupos docentes, con 1598 estudiantes y 53 profesores de las escuelas: Tania la Guerrillera, en Pinar del Río, Presidente Allende y Fulgencio Oroz en La Habana, René Fraga en Matanzas, Octavio García en Cienfuegos, Rafael Ma. de Mendive en Sancti Spíritus y Rita Longa en Las Tunas.

El Proyecto tiene la intención de dar "herramientas" a los estudiantes para que ellos puedan regular y autorregular su comportamiento, su comunicación con los demás; para motivarlos de manera que sepan reflexionar y orientarse acertadamente ante su realidad.

Las principales barreras que se han tenido que vencer para producir el cambio son: La costumbre, la inercia del pensamiento, el hábito entronizado de que todo lo que hacemos se nos indica desde un nivel superior, y por tanto es homogéneo, pero la realidad es extremadamente diversa.

Se logró hacer del diagnóstico la verdadera guía para su concreción en cada grupo y escuela. Aprender a hacer en cada lugar lo que se requiere para producir la transformación con la participación de todos y cada uno, será una contribución a la educación cubana.

La metodología del Proyecto, correctamente utilizada, contribuye a que los estudiantesaprendan la cultura de la participación en la dinámica escolar porque se prioriza el trabajo en equipo, la argumentación, la escucha activa respetuosa entre estudiantes y entre estos y sus profesores. La participación es gestada, estimulada y consolidada por una acción consciente y sistemática, con el empleo de métodos pedagógicos apropiados, propiciadores de lasocialización en espacios institucionales democráticos. Aprender a resolver los problemas existentes movilizando las energías, vivencias, experiencias... de todos los integrantes del grupo. Este puede considerarse el aprendizaje principal.

El elevado nivel de satisfacción manifestado por los participantes es muy positivo y estimulante, constituye un indicador de gran importancia para los que participan en un Proyecto de estas características y que no siempre se tiene en cuenta. Pero ello no es suficiente, es necesario ver su influencia en la solución de los problemas, en la transformación de las personas, de su manera de pensar y actuar, en los cambios que tienen lugar en los sentimientos, pensamientos y conductas de los participantes.

Es el inicio de un proceso y se aprecia que el proyecto ha provocado ciertos cambios en las conductas comunicativas, afectivas y sociales en los estudiantes y profesores implicados. 
Los estudiantes expresan que el Proyecto les aporta en que: (Se colocan textualmente las frases expresadas, solo se agrupan según las unidades de análisis).

- Debaten temas cercanos a su edad, que les interesan. Les despierta interés, los anima, los motiva y saca de la monotonía. Propicia la participación, es dinámico. La utilización de técnicas participativas. Su realización en espacios abiertos, fuera del aula. Pueden hablar, ser sinceros. Se puede expresar libremente lo que se piensa. Sin miedo a equivocarse.

- Les permite compartir ideas, inquietudes y opiniones. Facilita el fortalecimiento de valores.Los ayuda a tener una nueva visión de la realidad. Permite enfrentar la crisis de valores, de sentimientos.

- Trata de mejorar su forma de ser y actuar. Los enseña a autoanalizarse, a reflexionar cómo nos vemos y somos. Enseña a cómo comportarse. Aprenden a controlarse. Ayuda a vencer obstáculos Transforma su carácter. Permite identificar lo que hay que cambiar. Proporciona herramientas para el cambio, para la transformación.

- Coloca al ser humano en primer plano. Enseña a ponerse en lugar del otro, a cómo tratara los demás. Promueve la voluntad de servir.

- Promueve la coeducación. Estimula las relaciones, a unirnos y ayudarnos. A darse cariño. Solidaridad.

- Mejoran como persona. Los prepara e instruye para su profesión. Les ofrece un modelo decómo trabajar con los niños. Logran trasmitir las enseñanzas a los demás. Brinda enseñanzas para la vida.

\section{CONCLUSIONES:}

En las condiciones actuales es imprescindible que las instituciones que forman maestros reconozcan la significación de la orientación histórico-cultural en el proceso de educación de la personalidad de los estudiantes y presten especial atención a la subjetividad de los adolescentes que se forman en ellas.

La valoración realizada puso de manifiesto que el proyecto está siendo exitoso y motivador, considerando que hay cuatro razones fundamentales del éxito:

1. La metodología participativa utilizada, con los estudiantes como actores.

2. La selección de los mensajes para los temas que se llevaron a los talleres por sucercanía a las necesidades e intereses de los estudiantes.

3. El dominio y comprensión que han ido alcanzando los profesores coordinadores sobrelos contenidos de los temas a trabajar y la metodología.

4. La pasión que le imprimen los coordinadores para mejorar la calidad de la formaciónde los 
futuros maestros.

De acuerdo con la experiencia obtenida el Proyecto Con infinito amor: La educación del carácter y los valores familiares, resulta útil para la formación de los futuros maestros. 


\section{BIBLIOGRAFÍA}

Fariñas León, Gloria. (2009) Psicología, Educación y Sociedad. Un estudio sobre eldesarrollo humano. La Habana, Cuba. Editorial Pueblo y Educación.

Freire, Pablo. Pedagogía del oprimido. Grupo editorial Siglo XXI, Buenos Aires, 2013.

MINED. Normativas e indicaciones metodológicas y de organización para el desarrollo deltrabajo en las escuelas pedagógicas. Volumen III, curso escolar 2017 - 2018

Mendoza Portales, Lissette y otros. Prácticas cubanas para educar en valores y laconvivencia ciudadana. La Habana, 2013

Minujin, Alicia y otros. Sistema autorregulado. Material mimeografiado, La Habana, 1994.Proyecto Con infinito amor. Memorias de los 5 talleres nacionales. La Habana, 2014-18. Preguntar, dudar y opinar: Taller. En; Boletín Diálogo No. 33, La Habana, 2013.

\begin{tabular}{|c|c|}
\hline Anexo No. 1 Plan temático. & $\begin{array}{l}\text { Anexo No. } 2 \text { Sistema de acciones que guían el } \\
\text { Proyecto }\end{array}$ \\
\hline $\begin{array}{l}\text { I. Educación del carácter } \\
\text { 1. Educación del carácter. } \\
\text { 2. Modelos (ideales) } \\
\text { 3. Valores universales y las metasen la vida. } \\
\text { 4. Causas y resolución de conflictos. } \\
\text { 5. Diversidad humana. } \\
\text { II. Educación de valores familiares } \\
\text { 1. La familia como la escuela del amor. } \\
\text { 2. Preparando a la juventud para formar } \\
\text { familia. } \\
\text { 3. Maternidad y paternidad responsable. } \\
\text { 4. Comunicación. } \\
\text { 5. Métodos educativos. } \\
\text { III. Educación para el amor y la sexualida } \\
\text { 1. El amor y la sexualidad responsable. } \\
\text { 2. Adicciones. } \\
\text { 3. Infecciones de transmisión sexual. } \\
\text { 4. Embarazo en la adolescencia. }\end{array}$ & $\begin{array}{l}\text { 1. Creación o reorganización de los Gruposcoordinadores. } \\
\text { 2. Selección nuevos de profesores } \\
\text { coordinadores. } \\
\text { 3. Información inicial y sistemática al Consejode Dirección } \\
\text { de cada escuela. } \\
\text { 4. Preparación } \\
\text { coordinadores. } \\
\text { 5. Selección de los grupos. } \\
\text { 6. Preparación de los profesores guías. } \\
\text { 7. Diagnóstico. (Complemento del ler taller). } \\
\text { 8. Elaboración del cronograma de los talleres.Incorporado al } \\
\text { Sistema de trabajo escolar. } \\
\text { 9. Ejecución de los talleres planificados. } \\
\text { 10. Registro del diseño de cada taller. } \\
\text { 11. Organización } \\
\text { complementarias extradocentes y extraescolar. } \\
\text { 12. Recogida de evidencias. } \\
\text { 13. Información en las reuniones sindicales. } \\
\text { 14. Cierre de la etapa. } \\
\text { sistematización. Evaluación }\end{array}$ \\
\hline
\end{tabular}

